\title{
Los materiales didácticos digitales en educación infantil: análisis de repositorios institucionales
}

\author{
Vicente Gabarda Méndez ${ }^{1}$; ; Nerea Rodríguez Regueira ${ }^{2}$; ; Carlos González Ruiz ${ }^{3}$ (C) \\ ${ }^{1}$ Universidad de Valencia (UV), España; ${ }^{2}$ Universidad de Santiago de Compostela (USC), España; ${ }^{3}$ Universidad de La \\ Laguna (ULL) / Universidad Internacional de la Rioja (UNIR), España
}

Resumen. Las administraciones educativas autonómicas han puesto en marcha, dentro de la digitalización de los procesos de enseñanza y aprendizaje, la creación de portales institucionales donde se incorporan repositorios de materiales didácticos que pretenden servir de apoyo a los docentes de las diferentes etapas educativas, bajo el argumento del trabajo colaborativo. Bajo este planteamiento, el presente trabajo tiene por objetivo analizar una selección de recursos para la etapa de Educación Infantil que se encuentran alojados en los repositorios institucionales de Canarias, Galicia y Comunidad Valenciana. Concretamente, el análisis se centra en aspectos como el tipo de recurso, el idioma en que se ofrece, el área que se trabaja, la edad recomendada, la autoría, el año de creación o el tipo de licencia, tratando de ofrecer una descripción comparativa sobre la disponibilidad de materiales entre los distintos territorios. Los resultados arrojan que los materiales son, mayoritariamente, objetos digitales de aprendizaje, desarrollados en lengua castellana (aunque con presencia notable de lenguas cooficiales) por docentes a nivel individual y sin actualizaciones recientes. Además, se centran especialmente en las áreas de conocimiento del entorno y los lenguajes, pero no son específicos en relación a la edad de los destinatarios.

Palabras clave: material didáctico; educación básica; digitalización; educación infantil; metadatos.

Materiais didáticos digitais na educação infantil: análise de repositórios institucionais

Resumo. Como parte da digitalização do processo de ensino-aprendizagem, as administrações das comunidades autônomas da Espanha iniciaram a criação de portais institucionais onde incorporam repositórios de materiais didáticos destinados a apoiar os professores das diferentes etapas educacionais, dentro de uma perspectiva de trabalho colaborativo. Com base nesta abordagem, este trabalho visa analisar uma seleção de recursos para a etapa da Educação Infantil armazenados nos repositórios institucionais das IIhas Canárias, Galiza e Valência. Especificamente, a análise enfoca aspectos como o tipo de recurso, o idioma em que é oferecido, a área trabalhada, a idade recomendada, a autoria, o ano de criação ou o tipo de licença, tentando oferecer uma descrição comparativa sobre a disponibilidade de materiais entre os diferentes territórios. Os resultados demonstram que os materiais são, em sua maioria, objetos de aprendizagem digitais, elaborados em espanhol (embora com uma presença notável de línguas cooficiais), por professores de forma individual e sem atualizações recentes. Além disso, concentram-se especialmente nas áreas de conhecimento ambiental e linguístico, mas não são específicos quanto à idade dos destinatários.

Palavras-chave: material didático; educação básica; digitalização; educação infantil; metadados.

Digital teaching materials in early childhood education: analysis of institutional repositories Abstract. Regional educational administrations have launched, within the digitization of teaching and learning processes, the creation of institutional portals that include repositories of materials for teaching whose goal is to support teachers of the different stages, under the collaborative work's argument. Under this approach, this study aims to analyse a selection of resources for Early Childhood Education that are included in the institutional repositories of Canarias, Galicia and Comunidad Valenciana. Specifically, the analysis focuses on aspects such as the type of resource, the language in which it is offered, the area being worked on, the recommended age, the authorship, the year of creation or the type of license, trying to provide a comparative description of the availability of materials between the different territories. The results show that the materials are, for the most part, digital learning objects, developed in the Spanish language (with an important presence of the regional languages) by individual teachers and without recent updates. In addition, they focus especially on the areas of knowledge of the environment and languages, but are not specific in relation to the age of the students.

Keywords: teaching material; basic education; digitization; early childhood education; metadata. 


\section{Introducción}

No cabe duda de que los materiales y recursos que se ponen al servicio de los procesos de enseñanza y aprendizaje son una cuestión clave para el diseño, implementación y evaluación de los procesos formativos. Forman parte, junto a cuestiones como las habilidades docentes o las metodologías, de algunos elementos básicos que determinan el modo en que se desempeñan estos procesos y que condicionan la calidad con que se implementan.

Estos materiales pueden tener formatos diversos, especialmente teniendo en cuenta que en las últimas décadas están pasando por un proceso de metamorfosis ligada a la digitalización de los recursos. De esta forma, los tradicionales libros de texto, han pasado a convertirse en recursos digitales que tratan de adaptarse a los nuevos escenarios de aprendizaje y a los intereses y necesidades de los destinatarios en nuestra actual realidad social.

En la etapa de Educación Infantil, contexto en el que se centra la presente investigación, el abordaje del estudio de los materiales puede resultar controvertida, dada la variedad de enfoques desde la que puede abordarse la acción formativa y las características de los sujetos de aprendizaje. A este fenómeno hay que añadir, además, la integración de las Tecnologías Digitales (en adelante, TD), que ha contribuido, en este caso, a multiplicar las posibilidades para la elaboración de materiales didácticos digitales (Bus, Neuman y Roskos, 2020).

En esta misma línea, cabe apuntar que la Administración central, así como las diferentes Administraciones autonómicas, han tratado de potenciar, a través de diferentes estrategias, esta integración de las TD en el ámbito educativo y han planificado y ejecutado acciones vinculadas al tratamiento de los recursos didácticos. Una de ellas es la creación de repositorios institucionales donde se alojan materiales de diferentes etapas educativas, contribuyendo a crear una cultura de colaboración y de construcción conjunta de conocimiento (Sanabria, Álvarez y Peirats, 2017).

Toda esta realidad sirve de marco general para el planteamiento de nuestro trabajo que se centrará, precisamente, en el análisis de una selección de los materiales que están alojados en estos repositorios institucionales, poniendo el foco en el segundo ciclo de Educación Infantil y específicamente en los repositorios de Canarias, Galicia y Comunidad Valenciana. 


\section{Los repositorios institucionales como iniciativa gubernamental}

Como avanzábamos en el punto anterior, en la actualidad, la mayor parte de las comunidades autónomas de España cuentan con una estrategia para la integración de las TD en los centros educativos de las diferentes etapas formativas. Este es también el caso de las Comunidades Autónomas a las que se refiere este artículo que, entre otras estrategias, contemplan la creación de portales y plataformas institucionales de materiales y recursos multimedia para la docencia (Sanabria, Álvarez y Peirats, 2017).

Diversos estudios han tratado, durante las dos últimas décadas, de analizar desde perspectivas diversas las características y funcionalidades de estos repositorios y portales educativos (Area, 2003; Gertrudix, 2006; López, 2007; Martínez, Martínez y López, 2012), Ilegando a algunas consideraciones que ayudan a comprender la idiosincrasia de estas herramientas:

- Se trata de sitios web que cumplen una función informativa (ofrecen datos de interés para los destinatarios) y formativa (incluyen recursos orientados hacia la mejora de las competencias de los usuarios).

- Pueden considerarse espacios de almacenamiento de información y documentación: materiales didácticos, noticias, espacios de colaboración, etcétera.

- Funcionan bajo el principio de colaboración, siendo la clave de su dinámica que los usuarios (en este caso docentes de las diferentes etapas) compartan recursos elaborados de manera individual o colectiva.

Más reciente y vinculado con nuestro trabajo es el estudio de Santana, Eirín y Suelves (2017), quienes realizan una comparación entre los tres portales que servirán de contexto en el presente estudio: EspazoAbalar, de Galicia, EcoEscuela 2.0. de Canarias y Mestre a Casa, de Comunidad Valenciana. El análisis, que se centra en las dimensiones tecnológica, pedagógica y sociocomunicativa, concluye que no existen diferencias notables entre los tres portales y que los puntos débiles son compartidos (especialmente relacionados con la estructura de los portales y la falta de actualización). Sin embargo, apuntan a la potencialidad que tienen estas herramientas como repositorio para los materiales a los que puede acceder el profesorado para el desempeño de su labor docente.

Otra aproximación al estudio de este tipo de portales es la propuesta de Chirino, Romero, Castro y Etopa (2018), quienes realizan un análisis del Portal Procomún y del Portal Educativo de Canarias. Estos autores concluye- 
ron que el diseño de los portales ha mejorado, habiendo una gran diversidad de materiales que necesitaban ser actualizados. Asimismo, destacaron el potencial de estos recursos como repositorios de materiales, aunque contaban con la debilidad de no ofrecer una guía de ayuda al profesorado para cambiar su metodología de enseñanza. Por último, apuntaban a la necesidad de fomentar la interacción entre docentes en dichos espacios como clave de su actualización constante y la mejora de su dinámica.

Por otro lado, Peirats, Eirín y Rodríguez (2019) realizaron un estudio sobre las visiones de los agentes implicados en el diseño, difusión y utilización de los materiales en los portales educativos de Galicia y Comunidad Valenciana. Dicho estudio se centró en el alumnado, profesorado y familias, destacando las siguientes cuestiones: el profesorado y las familias afirman que es fundamental hacer uso de los recursos por diferentes razones, a destacar, la omnipresencia de las TD en la sociedad actual. Por otra parte, el alumnado comenta que le gusta trabajar tanto con materiales clásicos (libros de texto), como con los materiales ya citados. Como dispositivo para trabajar en la escuela prefieren el ordenador, frente a los móviles, tabletas, etc.

\section{La producción de materiales digitales para la docencia}

Apuntábamos anteriormente que los materiales didácticos han ido evolucionando en los últimos años a raíz de la integración de las TD en los procesos formativos y por los procesos de digitalización que se derivan de ella.

Una de las cuestiones clave a este respecto es quién suele asumir la responsabilidad en la creación de los materiales. En los últimos estudios realizados en el ámbito nacional (Cepeda, Gallardo y Rodríguez, 2017; González Ruiz y Chirino, 2019; Sanabria, Álvarez y Peirats, 2017), se ha podido comprobar que son los docentes en activo los que normalmente crean los recursos, principalmente a través de dos modalidades: a través del consumo o adaptación de los recursos provenientes para su docencia, una tesis que también refrenda el estudio de Fernández y Rodríguez (2017).

Otro estudio que destaca en este sentido es el realizado por González, Martín y Vega (2018), quienes realizaron un trabajo sobre el proceso de creación, autorías y desarrollo de materiales en el Portal Procomún y del Portal Educativo de Canarias. En dicho estudio, se refrendaba la idea de que la mayor parte de los materiales están creados por los equipos docentes en activo en las diferentes etapas educativas. Una cuestión que queda por resolver, sin embargo, es cómo se produce el proceso de creación de materiales y, por tanto, cuestiones como quiénes participan de manera directa e 
indirecta, si parten de un análisis de necesidades o si posteriormente son evaluados, tal y como se propone en Procomún. También en esta línea, es muy relevante destacar que el cambio de formato de los materiales requiere considerar un nuevo modo de evaluarlos, siendo necesario tener en cuenta, como propone Area (2017), cinco dimensiones: la tecnológica, la pedagógica, la funcionalidad docente, la funcionalidad discente, y los procesos de producción, distribución y consumo.

En base a todo lo expuesto, el objetivo principal de este estudio es analizar las principales características descriptivas de los materiales didácticos alojados en los repositorios institucionales de Canarias, Comunidad Valenciana y Galicia. De este modo, se abordan cuestiones como el idioma en que está elaborado el recurso, el año de creación, el tipo de material que es, la autoría, la edad recomendada, el área de Educación Infantil con que se vincula y el tipo de licencia de uso. Complementariamente, se exploran las diferencias entre los materiales de las diferentes Comunidades Autónomas en base a los diferentes criterios de análisis.

\section{Método y materiales}

Este artículo se basa en un estudio múltiple de casos a través de la compilación de casos individuales. Tal y como establecen Strauss y Corbin (1998), esta comparación requiere, entre otros aspectos, compartir características similares que, en este caso, serían: un marco teórico común, la utilización de un mismo instrumento y el mismo procesamiento de análisis de la información.

En relación a este último aspecto, se ha empleado la metodología de análisis de contenido entendida como "el conjunto de técnicas de análisis comunicativas usando procedimientos sistemáticos y objetivos para describir los contenidos de los mensajes" (Bardin, 2004, p. 29). Cabe señalar, que la elección de la misma también se justifica al considerar que este es uno de los enfoques más sólidos en cuanto al análisis de materiales didácticos se refiere y materializado en múltiples investigaciones (Richaudeau, 1981; Parcerisa, 1996; Castiello, 2002; López-Gomez, 2017)

La muestra está compuesta por materiales didácticos digitales cuyo target es el alumnado de Educación infantil y albergados en los repositorios institucionales de tres Comunidades Autónomas: EspazoAbalar (Galicia), Mestre a Casa (Comunidad Valenciana) y EcoEscuela 2.0. (Canarias). 
El objetivo principal del presente artículo es ofrecer una descripción comparativa sobre la disponibilidad de materiales entre los distintos territorios considerando la diversidad de la oferta digital actual para esta etapa educativa.

\subsection{Muestra}

Cabe comenzar exponiendo que cada repositorio presenta una oferta diferente respecto a la cantidad de materiales y su tipología. En nuestro caso, se ha procedido a seleccionar un total de 45 materiales (15 por cada repositorio), pertenecientes a las plataformas institucionales EspazoAbalar (Galicia), Mestre a Casa (Valencia) y EcoEscuela 2.0 (Canarias).

La selección de los materiales se ha realizado en base a los siguientes criterios:

- Ubicación: Se han seleccionado materiales que formen parte de los repositorios institucionales de las Comunidades Autónomas anteriormente señaladas. En la tabla 1 se muestra una distribución del número de materiales identificados por cada una de las plataformas.

- Destinatarios: se trata de materiales cuyos destinatarios sea alumnado escolarizado en el segundo ciclo de Educación Infantil

- Áreas curriculares: se han analizado materiales de las diferentes áreas en las que se estructura la etapa de Educación Infantil: 1) Conocimiento de sí mismo y autonomía personal; 2) Conocimiento del entorno; 3) Lenguajes: Comunicación y representación.

- Adecuación: se ha contemplado como requisito que se puedan analizar todas las dimensiones contempladas en el instrumento de análisis que se describe en este trabajo: características identificadoras del material o recursos educativos, estructura del material, dimensión tecnológica, dimensión de diseño, dimensión pedagógica, dimensión de contenido, evaluación y seguimiento.

Tabla 1. Distribución de la muestra

\begin{tabular}{lcc}
\hline $\begin{array}{c}\text { Repositorio institucional y } \\
\text { Comunidad Autónoma }\end{array}$ & $\begin{array}{c}\text { Total de recursos para } \\
\text { el segundo ciclo de } \\
\text { Educación Infantil }\end{array}$ & $\begin{array}{c}N^{\circ} \text { de recursos } \\
\text { analizados }\end{array}$ \\
\hline EspazoAbalar (Galicia) & 81 & 15 \\
Mestrea Casa (Comunidad Valenciana) & 100 & 15 \\
EcoEscuela 2.0. (Canarias) & 270 & 15 \\
Total & 451 & 45
\end{tabular}

Fuente: Elaboración propia. 
Como puede observarse, el total de materiales que se aloja en los diferentes repositorios es diverso. Sin embargo, a fin de ofrecer una panorámica más homogénea, se analizan un total de 45 recursos, que constituyen en torno a una décima parte de los materiales alojados en los diferentes repositorios.

\subsection{Instrumento}

Para el desarrollo del instrumento considerado en esta investigación se adaptó el instrumento "Guía para el análisis de las plataformas y/o portales web" del Proyecto Escuel@ Digit@।, cuyas características pueden consultarse en el trabajo de Cepeda, Gallardo y Rodríguez (2017) y que se detallan a continuación. Cabe destacar, no obstante, que, aunque esta guía estaba centrada en el análisis de materiales de la etapa de Educación Primaria, se han realizado algunas adaptaciones para dar respuesta a las características e idiosincrasia de la Educación Infantil

La guía contempla un total de nueve variables de análisis con objeto de dar una respuesta global al estudio de las características de los materiales:

- Datos de identificación del evaluador: se identifica el autor de la evaluación del material.

- Datos de identificación del material o recurso educativo: comprende la información descriptiva del recurso, atendiendo a aspectos como su denominación, tipología y autoría.

- Estructura del material: se ofrece una descripción general del material desde un punto de vista organizativo, atendiendo a las secciones que lo componen y su contenido específico.

- Dimensión tecnológica: se analizan cuestiones como la navegabilidad, la accesibilidad, las plataformas en que puede utilizarse o la interactividad.

- Dimensión del diseño: se centra en desentrañar las características funcionales y de diseño más destacables, teniendo en cuenta las características evolutivas de los destinatarios.

- Dimensión pedagógica: se analiza el material desde un punto de vista didáctico considerando el tipo de actividades, la organización del contenido o las estrategias metodológicas, entre otros aspectos.

- Dimensión de contenido: se centra en el estudio de cuestiones como la presencia de diversidad, la representación de los avatares o el modo en que se organizan y tratan los contenidos en función de la etapa educativa. 
- Evaluación y seguimiento: se analiza el tratamiento que le da el material a la evaluación (presencia de criterios de evaluación, estrategias evaluativas, feedback, etc.).

- Comentarios finales: se trata de un espacio para la evaluación complementaria, así como para la identificación de las principales ventajas e inconvenientes del material.

En el marco de nuestro trabajo, se va a centrar especialmente la atención sobre los datos de identificación del material, dotando al estudio de un carácter descriptivo sobre cuestiones de carácter específico que se detallarán en el siguiente apartado.

\subsection{Procedimiento}

Tal y como se apuntaba anteriormente, esta investigación propone el análisis de 45 materiales ( 15 por cada repositorio institucional) desde un punto de vista descriptivo de sus datos de identificación. Concretamente, se exploran las siguientes variables:

- Idioma: teniendo en cuenta que dos, de las tres Comunidades Autónomas que son objeto de análisis, tienen lengua cooficial y que el contexto de análisis son plataformas institucionales donde se fomenta su utilización, podemos encontrar recursos en castellano, gallego, valenciano y otras lenguas curriculares como el inglés.

- Tipo de material: a fin de clasificar los recursos, se ha tomado en consideración la propuesta de Area (2017) para la tipología de materiales didácticos:

Objeto digital. Es un archivo digital que porta contenido, información y/o conocimiento. Cuando están almacenados de forma organizada constituyen un repositorio de objetos digitales.

- Objeto digital de aprendizaje. Es un tipo particular de objetos digitales creados con intencionalidad didáctica a corto plazo, que implican alguna acción del estudiante. Adoptan, en la mayor parte de las ocasiones, el formato de actividades o ejercicios aislados.

- Material Didáctico Digital (MDD). Es un paquete estructurado didácticamente de objetos digitales en línea, dirigido a facilitar al alumnado el desarrollo de experiencias de aprendizaje en torno a una unidad de saber o competencia. Son materiales para la educación formal, ya que en los mismos subyace una propuesta o proyecto de desarrollo curricular. Este tipo de materiales educativos tienen una amplia y larga tradición en nuestros estudios y ya conocemos 
muchas de sus dimensiones de análisis producidos en soporte de papel (libros de texto) o multimedia (CD-ROM, DVD y similares).

- Materiales profesionales de docentes. Son el conjunto de objetos digitales que ofrecen programaciones, experiencias prácticas, propuestas elaboradas de intervenciones educativas, espacios de publicación del profesorado (blogs, wikis). Son recursos de interés para el profesorado en su autoformación y mejora profesional.

- Apps, herramientas y plataformas online. Es software. A veces son de propósito general y en otras ocasiones específicamente creados para el ámbito educativo. Hay cientos y constantemente están en evolución y crecimiento (p. 20-21).

- Área de educación infantil que se trabaja: respondiendo a las directrices recogidas en el Real Decreto 1630/2006, de 29 de diciembre, por el que se establecen las enseñanzas mínimas del segundo ciclo de Educación infantil, se identifican tres áreas: 1) Conocimiento de sí mismo y autonomía personal; 2) Conocimiento del entorno; 3) Lenguajes: Comunicación y representación.

- Edad recomendada: dado que el análisis se centra en el segundo ciclo de Educación Infantil, las edades recomendadas en los materiales oscilan entre los tres y los seis años, pudiendo tratarse de un recurso de carácter global (3-6 años) o para una edad específica (3 años, 4 años, 5 años, 6 años). En alguna Comunidad, esta variable se identifica con el curso $\left(4^{\circ}, 5^{\circ} \circ 6^{\circ}\right.$ de Educación Infantil).

- Año de creación: a fin de identificar la actualización de los materiales, se recoge el año de creación o, en su caso, el año en que se aloja en el repositorio institucional correspondiente.

- Tipo de licencia: se analiza el tipo de licencia utilizada que van desde la más restrictiva en cuanto a condiciones de uso y divulgación a la más permisiva. Hemos considerado, por lo tanto: el Copyright (implica que ninguna obra puede ser copiada, distribuida, comunicada públicamente ni transformada sin permiso del autor), Copyleft (permite usar, modificar, copiar y distribuir un recurso siempre que se use el mismo tipo de licencia), Creative Commons (este tipo de licencia de uso libre, existen seis variantes, permite al autor decidir sobre las condiciones de copia, modificación y/o tipos de uso)

- Autoría: se analiza si se trata de un recurso elaborado por un docente a nivel individual, un grupo de docentes, una entidad privada o un recurso creado por la propia Administración Autonómica o Nacional en el contexto de algún proyecto específico. 
Estas variables nos permiten, por tanto, explorar cuestiones básicas que ofrecen un panorama acerca de los materiales alojados en cada uno de los repositorios, permitiendo, igualmente, una comparación de la idiosincrasia de éstos en cada uno de los territorios.

\section{Resultados}

Atendiendo al objetivo principal del trabajo, podemos afirmar que los 45 materiales analizados presentan algunos rasgos comunes, tal y como puede observarse en la tabla 2. En ella, se puede observar la predominancia del castellano como idioma $(64,4 \%)$, algo lógico si tenemos en cuenta que es la lengua oficial en una de las Comunidades y cooficial en las otras dos. Por otro lado, la mayor parte de los materiales son como objetos digitales de aprendizaje $(84,4 \%)$ que se centran en el desarrollo de un área específica (el $35,6 \%$ de ellos en el conocimiento del entorno y el mismo porcentaje, en el área de Lenguajes), siendo menos habituales los materiales de carácter globalizado. Por otro lado, no se especifica de forma clara la edad concreta para los destinatarios, asociándose a una etiqueta general de "Educación Infantil" para estudiantes de 3 a 5 años. En relación a la autoría, los materiales están elaborados por docentes a nivel individual $(37,8 \%)$ y con licencias Copyright o Creative Commons ( $50 \%$ en cada caso), poniendo de relieve que, aunque se comparten en repositorios institucionales, se limita en ocasiones la gestión de los recursos.

Tabla 2. Características generales de los materiales analizados

\begin{tabular}{llrr}
\hline \multicolumn{1}{c}{ Característica } & \multicolumn{1}{c}{ Categorías } & $\%$ \\
\hline Idioma & Castellano & 29 & 64,4 \\
& Gallego & 7 & 15,6 \\
& Valenciano & 5 & 11,1 \\
Tipología & Inglés & 4 & 8,9 \\
& Objeto digital de aprendizaje & 38 & 84,4 \\
& Material profesional docente & 3 & 6,7 \\
Área & Material didáctico digital & 4 & 8,9 \\
& Conocimiento de sí mismo & 8 & 17,8 \\
& Conocimiento del entorno & 16 & 35,6 \\
\multirow{2}{*}{ Edades recomendadas } & 16 & 35,6 \\
& Lenguajes & 5 & 11,1 \\
& Globalizado & 2 & 4,4 \\
& 3 años & 0 & 0 \\
& 4 años & 16 & 35,6 \\
& 5 años & 1 & 2,2 \\
& 3-4 años & 5 & 11,1 \\
& $4-5$ años & 21 & 46,7
\end{tabular}




\begin{tabular}{llrr}
\hline \multicolumn{1}{c}{ Característica } & \multicolumn{1}{c}{ Categorías } & $N$ & $\%$ \\
\hline Año de creación & 2007 & 2 & 4,4 \\
& 2011 & 4 & 8,8 \\
& 2012 & 2 & 2,2 \\
2013 & 3 & 3,3 \\
& 2014 & 12 & 26,7 \\
& 2015 & 6 & 13,3 \\
& 2016 & 1 & 2,2 \\
Tipo de licencia & No se especifica & 15 & 33,3 \\
& Copyright & 15 & 33,3 \\
& Copyleft & 0 & 0,0 \\
Autoría & Creative Commons & 15 & 33,3 \\
& No se especifica & 15 & 33,3 \\
& Docente individual & 17 & 37,8 \\
& Docente colectivo & 4 & 8,8 \\
& Proyecto de la administración & 5 & 11,1 \\
& Empresa privada & 4 & 8,8 \\
& No se especifica & 15 & 33,3
\end{tabular}

Fuente: Elaboración propia.

Si abordamos las posibles diferencias entre las diferentes Comunidades Autónomas en relación a estas mismas variables, hay aspectos muy reseñables.

En el caso de Canarias, la práctica totalidad de los materiales analizados están en lengua castellana (93,3\%), habiendo un único material en inglés. Atendiendo a la tipología, el 73,3\% son objetos digitales de aprendizaje, estando orientados al desarrollo de las diferentes áreas: conocimiento de sí mismo $(26,7 \%)$, conocimiento del entorno $(20,0 \%)$, lenguajes $(26,7 \%)$ y algunos de carácter globalizado (26,7\%). En relación a las edades recomendadas para su utilización, están dirigidos en su mayoría a los estudiantes de toda la etapa (3-6 años) independientemente de la edad (46,7\%) o dirigido a los de 5 años $(35,6 \%)$. Se trata de materiales desarrollados, de manera mayoritaria, en el año $2014(80,0 \%)$ y son recursos elaborados por docentes a título individual $(60,0 \%)$, aunque hay también un porcentaje reseñable que están elaborados por empresas (26,7\%). Por último, su licencia de explotación es, en general, de Copyright (86,7\%).

Tabla 3. Características de los materiales del repositorio EcoEscuela 2.0. (Canarias)

\begin{tabular}{llrc}
\hline \multicolumn{1}{c}{ Característica } & \multicolumn{1}{c}{ Categorías } & N & Porcentaje \\
\hline Idioma & Castellano & 14 & 93,3 \\
& Inglés & 1 & 6,7 \\
Tipología & Objeto digital de aprendizaje & 11 & 73,3 \\
& Material didáctico digital (MMD) & 4 & 26,7
\end{tabular}




\begin{tabular}{|c|c|c|c|c|}
\hline Característica & \multicolumn{2}{|c|}{ Categorías } & $\mathrm{N}$ & Porcentaje \\
\hline \multirow[t]{4}{*}{ Área } & \multicolumn{2}{|c|}{ Conocimiento de sí mismo } & 4 & 26,7 \\
\hline & \multicolumn{2}{|c|}{ Conocimiento del entorno } & 3 & 20,0 \\
\hline & \multicolumn{2}{|c|}{ Lenguajes } & 4 & 26,7 \\
\hline & \multicolumn{2}{|l|}{ Globalizado } & 4 & 26,7 \\
\hline \multirow{6}{*}{$\begin{array}{l}\text { Edades recomen- } \\
\text { dadas }\end{array}$} & \multicolumn{2}{|l|}{3 años } & 1 & 6,7 \\
\hline & \multicolumn{2}{|l|}{4 años } & 0 & 0,0 \\
\hline & \multicolumn{2}{|l|}{5 años } & 1 & 6,7 \\
\hline & \multicolumn{2}{|l|}{ 3-4 años } & 1 & 6,7 \\
\hline & \multicolumn{2}{|l|}{ 3-6 años } & 7 & 46,7 \\
\hline & \multicolumn{2}{|l|}{ 4-5 años } & 5 & 33,3 \\
\hline \multirow[t]{3}{*}{ Año de creación } & \multicolumn{2}{|l|}{2007} & 2 & 13,3 \\
\hline & \multicolumn{2}{|l|}{2014} & 12 & 80,0 \\
\hline & \multicolumn{2}{|l|}{2015} & 1 & 6,7 \\
\hline \multirow[t]{8}{*}{ Tipo de licencia } & \multicolumn{2}{|l|}{ Copyright } & 13 & 86,7 \\
\hline & \multicolumn{2}{|l|}{ CopyLeft } & 0 & 0,0 \\
\hline & \multirow[t]{6}{*}{ Creative Commons } & BY & 0 & 0,0 \\
\hline & & BY-NC & 0 & 0,0 \\
\hline & & BY-NC-SA & 1 & 6,7 \\
\hline & & BY-NC-ND & 1 & 6,7 \\
\hline & & BY-SA & 0 & 0,0 \\
\hline & & BY-ND & 0 & 0,0 \\
\hline \multirow[t]{4}{*}{ Autoría } & \multirow[t]{2}{*}{ Docente } & Individual & 9 & 60,0 \\
\hline & & Colectivo & 2 & 13,3 \\
\hline & \multicolumn{2}{|c|}{ Consejería / Proyectos públicos } & 0 & 0,0 \\
\hline & \multicolumn{2}{|l|}{ Empresa privada } & 4 & 26,7 \\
\hline
\end{tabular}

Fuente: Elaboración propia.

En el caso de la Comunidad Valenciana, los materiales analizados están elaborados de manera mayoritaria en castellano $(60,0 \%)$, aunque hay representación de la otra lengua cooficial del territorio, el valenciano (33,3\%). Al igual que en el caso de Canarias, son objetos digitales de aprendizaje (93,3\%), centrados especialmente en las áreas de conocimiento del entorno $(46,7 \%)$ y lenguajes $(40,0 \%)$. A pesar de que se catalogan por edades, el $66,7 \%$ de los materiales analizados se identifican para los sujetos de 3,4 y 5 años, estando el resto (33,3\%) orientados a estudiantes de 5 años.

Es reseñable que el repositorio de Comunidad Valenciana, no recoge información sobre el resto de variables, impidiendo conocer el año de creación de los materiales, su autoría o el tipo de licencia asociada. 
Tabla 4. Características de los materiales de Mestre a Casa (Comunidad Valenciana)

\begin{tabular}{llrr}
\hline \multicolumn{1}{c}{ Característica } & \multicolumn{1}{c}{ Categorías } & \multicolumn{1}{c}{ N } \\
\hline Idioma & Castellano & $9(60,0 \%)$ & 60,0 \\
& Valenciano & $5(33,3 \%)$ & 33,3 \\
& Inglés & $1(6,7 \%)$ & 6,7 \\
Tipología & Objeto digital de aprendizaje & $14(93,3 \%)$ & 93,3 \\
& Material profesional docente & $1(6,7 \%)$ & 6,7 \\
Área & Conocimiento de sí mismo & $2(13,3 \%)$ & 13,3 \\
& Conocimiento del entorno & $7(46,7 \%)$ & 46,7 \\
& Lenguajes & $6(40,0 \%)$ & 40,0 \\
Edades recomen- & 3-6 años & $10(66,7 \%)$ & 66,7 \\
dadas & 3 años & 0 & 0,0 \\
& 4 años & 0 & 0,0 \\
& 5 años & $5(33,3 \%)$ & 33,3 \\
& 6 años & 0 & 0,0 \\
Año de creación & No se especifica & $15(100 \%)$ & 100,0 \\
Tipo de licencia & No se especifica & $15(100 \%)$ & 100,0 \\
Autoría & No se especifica & $15(100 \%)$ & 100,0
\end{tabular}

Fuente: Elaboración propia.

En el caso de Galicia, los materiales en lengua gallega y en lengua castellana representan la práctica totalidad de los recursos analizados $(46,7 \%$ y $40,0 \%$ respectivamente). Se trata de objetos digitales de aprendizaje $(86,7 \%)$ orientados al abordaje del conocimiento del entorno (40,0\%) o los lenguajes (40,0\%). Los destinatarios de los materiales son, sobretodo, estudiantes de 5 años $(66,7 \%)$ y están elaborados por docentes a nivel individual $(53,3 \%) 0$ colectiva (13,3\%). Los materiales están creados, en su mayoría, entre 2011 y 2015 (93,3\%) y con una licencia Creative Commons BY-NC-SA (73,3\%).

Tabla 5. Características de los materiales de EspazoAbalar (Galicia)

\begin{tabular}{llrc}
\hline \multicolumn{1}{c}{ Característica } & \multicolumn{1}{c}{ Categorías } & N & Porcentaje \\
\hline Idioma & Castellano & 6 & 40,0 \\
& Gallego & 7 & 46,7 \\
& Inglés & 2 & 13,3 \\
Tipología & Objeto digital de aprendizaje & 13 & 86,7 \\
& Material profesional docente & 2 & 13,3 \\
Área & Conocimiento de sí mismo & 2 & 13,3 \\
& Conocimiento del entorno & 6 & 40,0 \\
& Lenguajes & 6 & 40,0 \\
Edad recomendada & 1 & 6,7 \\
& Globalizado & 4 & 26,7 \\
& 3-6 años & 1 & 6,7 \\
& 3 años & 0 & 0,0 \\
& 4 años & 10 & 66,7
\end{tabular}




\begin{tabular}{|c|c|c|c|c|}
\hline \multirow{2}{*}{$\begin{array}{l}\text { Característica } \\
\text { Año de creación }\end{array}$} & \multicolumn{2}{|c|}{ Categorías } & \multirow{2}{*}{$\begin{array}{l}N \\
4\end{array}$} & \multirow{2}{*}{$\begin{array}{c}\text { Porcentaje } \\
26,7\end{array}$} \\
\hline & \multicolumn{2}{|l|}{2011} & & \\
\hline & \multicolumn{2}{|l|}{2012} & 2 & 13,3 \\
\hline & \multicolumn{2}{|l|}{2013} & 3 & 20,0 \\
\hline & \multicolumn{2}{|l|}{2015} & 5 & 33,3 \\
\hline & \multicolumn{2}{|l|}{2016} & 1 & 6,7 \\
\hline \multirow[t]{8}{*}{ Tipo de licencia } & \multicolumn{2}{|l|}{ Copyright. } & 2 & 7,3 \\
\hline & \multicolumn{2}{|l|}{ CopyLeft } & 0 & 0,0 \\
\hline & \multirow[t]{6}{*}{ Creative Commons } & BY & 0 & 0,0 \\
\hline & & BY-NC & 0 & 0,0 \\
\hline & & BY-NC-SA & 11 & 73,3 \\
\hline & & BY-NC-ND & 0 & 0,0 \\
\hline & & BY-SA & 2 & 7,3 \\
\hline & & BY-ND & 0 & 0,0 \\
\hline \multirow[t]{4}{*}{ Autoría } & \multirow[t]{2}{*}{ Docente } & Individual & 8 & 53.3 \\
\hline & & $\begin{array}{l}\text { Grupos de docentes } \\
\text { y/o seminarios }\end{array}$ & 2 & 13.3 \\
\hline & \multicolumn{2}{|c|}{ Consejería / Proyectos públicos } & 5 & 33,3 \\
\hline & \multicolumn{2}{|l|}{ Empresa privada } & 0 & 0,0 \\
\hline
\end{tabular}

Fuente: Elaboración propia.

Para finalizar se ofrece a continuación una tabla resumen con las 74 características más comunes de los materiales de cada Comunidad Autónoma en base a las diferentes variables:

Tabla 6. Rasgos más comunes de los materiales por repositorio

\begin{tabular}{|c|c|c|c|}
\hline Criterio & EcoEscuela 2.0. & Mestre a Casa & EspazoAbalar \\
\hline Idioma & Castellano & Castellano & Gallego \\
\hline Tipología & $\begin{array}{l}\text { Objeto digital de } \\
\text { aprendizaje }\end{array}$ & $\begin{array}{l}\text { Objeto digital de } \\
\text { aprendizaje }\end{array}$ & $\begin{array}{l}\text { Objeto digital de } \\
\text { aprendizaje }\end{array}$ \\
\hline Área & $\begin{array}{l}\text { Conocimiento de sí } \\
\text { mismo / Lenguajes / } \\
\text { Globalizado }\end{array}$ & $\begin{array}{l}\text { Conocimiento del } \\
\text { entorno }\end{array}$ & $\begin{array}{l}\text { Conocimiento del } \\
\text { entorno / Lenguajes }\end{array}$ \\
\hline Edad recomendada & 3-6 años & 3-6 años & 5 años \\
\hline Año de creación & 2014 & - & 2015 \\
\hline Tipo de licencia & Copyright. & - & Creative commons \\
\hline Autoría & Docente individual & 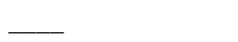 & Docente Individual \\
\hline
\end{tabular}

Fuente: Elaboración propia. 


\section{Discusión y conclusiones}

El análisis de los repositorios institucionales donde se alojan los materiales didácticos, nos ha permitido ver que hay elementos comunes entre ellos, como la finalidad con que se utilizan. Sin embargo, si analizamos desde un punto de vista comparativo los materiales de las tres comunidades autónomas, encontramos situaciones diversas;

- En relación al idioma, los materiales de las dos Comunidades Autónomas con lengua cooficial reconocida (Comunidad Valenciana y Galicia) suelen representar aproximadamente la mitad de los alojados en los repositorios, atendiendo tanto a la utilización de la propia lengua, como a los planes de plurilingüismo que se promueven desde las diferentes administraciones. Es reseñable, asimismo, que, en los tres casos, los materiales en inglés representan un porcentaje escaso.

- En las tres Comunidades Autónomas estos materiales son, en su mayoría, objetos digitales de aprendizaje. En este sentido, son objetos digitales con intencionalidad didáctica (actividades o ejercicios) orientados a la utilización directa por parte del estudiante. Este hecho representa que los materiales que se alojan están elaborados, de manera mayoritaria, por docentes a nivel individual, que comparten el trabajo que preparan para sus estudiantes.

- Las áreas de trabajo más habitual de los materiales en los tres contextos son el conocimiento del entorno y los lenguajes, aunque destaca la cantidad de materiales de carácter globalizado en Galicia. En este sentido, todos los materiales analizados responden a una o varias de las áreas que la normativa contempla para la Educación Infantil.

- En el caso de la edad, los materiales alojados en el repositorio gallego están orientados especialmente a 5 años, mientras que en el repositorio canario y el valenciano es más común encontrar materiales que pueden ser utilizados durante toda la etapa (3-6 años). Esta falta de definición dificulta una correcta utilización del material.

- En el caso de la Comunidad Valenciana, no es posible identificar el año de creación de los materiales, mientras que en el caso de Canarias y Galicia la mayor parte de los materiales datan de mediados de la década pasada. Estos datos ponen de manifiesto que los materiales están, en una gran parte, obsoletos, tanto en su forma como en el contenido. 
- El tipo de licencia más común de los materiales del repositorio de Canarias es Copyright, en el caso de Galicia es Creative Commons, mostrando dos posturas radicalmente opuestas en relación al uso de los materiales, o directamente, una ausencia de información, como es el caso de la Comunidad Valenciana.

Estos datos nos hacen reflexionar acerca de los cambios que implica una educación cada vez más digital y que lleva inherente una metamorfosis de los materiales didácticos (Area, 2017). Son cambios que van más allá del cambio de formato e implica transformaciones en su propia definición, en el papel del alumnado y el profesorado, así como en los procesos de creación, difusión y consumo de los mismos. Con el aumento exponencial de materiales digitales para la enseñanza y el aprendizaje se hizo necesario contar con espacios para albergarlos, clasificarlos y compartirlos. De forma más concreta, en el contexto español, la creación de estos espacios surgió a partir del compromiso de las Administraciones de proporcionar a la comunidad educativa un catálogo de recursos digitales que propiciarán la transformación de la escuela tradicional hacia la escuela digital (Baztán, 2014). En el caso que nos ocupa, los portales o plataformas objeto de estudio (EspazoAbalar, Mestre a Casa y EcoEscuela 2.0) son plataformas institucionales que dependen directamente de las administraciones educativas autonómicas (Consellería de Cultura, Educación e Universidade del Gobierno de Galicia; Conselleria d'Educació Cultura i Esport del Gobierno de Valencia y Consejería de Educación y Universidades del Gobierno de Canarias). Se trata de plataformas que funcionan como repositorios de materiales digitales de acceso gratuito y contienen recursos producidos por las propias administraciones o por los docentes, pudiendo acceder a los mismos docentes, familias u otros profesionales interesados.

El análisis realizado ha constatado una carencia en cuanto a la inclusión de metadatos o la correcta indexación de los materiales. Los metadatos deberían hacer una referencia adecuada al tipo de material, el área de conocimiento, los objetivos de aprendizaje, los requisitos técnicos y el nivel educativo o edad de los destinatarios. Este último aspecto es el menos cuidado en los materiales analizados. Los metadatos y la información que proporcionan resultan de vital importancia para que los docentes, u otros agentes educativos que accedan a los repositorios, puedan seleccionar adecuadamente los MDD. Tal y como señalan Pinto (2015) y Sánchez-Jara y Vega (2019), la información descriptiva de un material digital junto con la información técnica condicionan la selección de los materiales a la par que suponen un criterio de calidad de los mismos. Consideramos que, dado que la mayoría de CCAA disponen de un repositorio de MDD, sería muy recomendable el establecimiento de unos metadatos comunes que posibilitaría la portabilidad de unos repositorios a otros. 
También se ha corroborado el papel de los docentes como autores de los MDD analizados y motor de los repositorios, en consonancia con otros estudios como los de Sanabria, Álvarez y Peirats (2017) o González, Martín y Vega (2018). Este hecho sirve, además, para subrayar los cambios en la profesionalidad docente que se están dando en esta transformación hacia una escuela digital. Podemos observar cómo el profesorado está avanzando desde la posición de meros consumidores de contenidos a la de productores de los mismos, enfrentándose a lo que Montero y Vez (2003) denominan como "Síndrome del consumidor de productos culturales".

Cabe señalar, en este punto, que se han encontrado diferencias significativas entre los tres territorios en relación a la promoción de estrategias para la elaboración de materiales didácticos. De forma más concreta, únicamente en el caso concreto de Galicia, se ha constatado la promulgación de licencias de formación (convocadas cada año en el mes de junio) para la creación de lo que en la convocatoria denominan como "materiales curriculares digitales" que pueden ser elaborados por docentes individualmente 0 constituidos en grupos de trabajo (la última convocatoria se puede consultar en la "Resolución del 5 de junio de 2019, por la que se convocan licencias de formación para el curso 2019/2020 destinadas al funcionariado docente no universitario y se aprueban las bases para su concesión"). La peculiaridad de estas licencias es que los MDD creados deben pasar a formar parte del repositorio de contenidos del EspazoAbalar.

En resumen, los resultados han puesto de manifiesto que, aunque las diferentes administraciones tratan de promover iniciativas orientadas a la digitalización de los procesos de enseñanza y aprendizaje, los repositorios de materiales cuentan con algunas carencias muy reseñables que pueden condicionar su utilidad. Cuestiones como la falta de actualización de los materiales o la ausencia de especificaciones sobre la edad recomendada de los usuarios pueden desvirtuar su potencial como espacio de trabajo colaborativo. Consideramos básico, en este sentido, la propuesta de políticas, como la de Galicia, que promocionen la creación de contenido por parte de los docentes que, al margen de favorecer su competencia digital, sirva de impulso para la creación de un espacio actualizado de buenas prácticas.

Como futuras líneas de trabajo contemplamos el análisis de estos materiales desde un punto de vista técnico y pedagógico, que nos ayuden a tener una visión más holística de los recursos y poder evaluar, de esta manera, sus beneficios desde otra perspectiva. 


\section{Agradecimientos}

Este artículo forma parte del proyecto "Los materiales didácticos digitales en la Educación Infantil. Análisis y propuestas para su uso en la escuela y el hogar" (Infanci@ Digit@I: RTI2018-093397-B-I00), financiado por el Programa Estatal de I+D+i. Orientado a los Retos de la Sociedad.

\section{Referencias}

Area, M. (2003). De los web educativos al material didáctico web. Comunicación y Pedagogía, $188,32-38$.

Area, M. (2017). La metamorfosis digital del material didáctico tras el paréntesis Gutenberg. RELATEC: Revista Latinoamericana de Tecnología Educativa, 16(2), 13-28. https:// doi.org/10.17398/1695-288X.16.2.13

Bardin, L. (2004). Análise de conteúdo. 3. Lisboa: Edições.

Baztán, M. (2014). Los materiales didácticos digitales en la enseñanza no universitaria española. Análisis comparativo. Valencia: Ediciones Universitat Politécnica de València.

Bus, A.G., Neuman, S.B. y Roskos, K. (2020). Screens, Apps, and Digital Books for Young Children: The Promise of Multimedia. AERA Open. https://doi. org/10.1177/2332858420901494

Castiello, J.M. (2002). Los desafíos de la educación intercultural: migraciones y curriculum (Tesis doctoral). Universidad de Oviedo.

Cepeda, O., Gallardo, M.I. y Rodríguez, J. (2017). La evaluación de los materiales didácticos digitales. RELATEC: Revista Latinoamericana de Tecnología Educativa, 16(2), 7995. http://dx.medra.org/10.17398/1695-288X.16.2.79

Chirino, E., Romero, K.E., Castro, J.J. y Etopa, M.P. (2018). Análisis de portales con materiales didácticos digitales: Procomún y EcoEscuela 2.0. En: E. López, D. Cobos, A.H. Martín, L. Molina y A. Jaén (Coords). Experiencias pedagógicas e innovación educativa. Aportaciones desde la praxis docente e investigadora (pp.3161-3170). Madrid: Octaedro.

Fernández, R. y Rodríguez, J. (2017). The state of published research on teaching materials in Early Childhood Education. En E. Bruillard, A. Anichini \& G.-L. Baron (Eds.). Changing media - changing schools? 14th IARTEM Conference (pp. 122-126). Lisbon, IARTEM.

Gértrudix, F. (2006). Los portales educativos como fuentes de recursos materiales. Icono 14, 4(1). Recuperado de https://bit.ly/3mzXQGs

González, C.J. y Chirino, E. (2019). Análisis de materiales didácticos digitales ofertados desde un portal de contenidos abiertos: el caso de Canarias. Educar em Revista, 35(77), 19-36. https://doi.org/10.1590/0104-4060.68472

González, C.J., Martín, S. y Vega, A. (2018). Portales educativos: la producción de materiales didácticos digitales. @TIC, revista de innovación educativa, 20, 89-97.

López, R. (2007). Los portales educativos: clasificación y componentes. Anales de documentación, 10, 233-244. 
López-Gómez S. (2017) Análise descritiva e interpretativa do deseño e contido dos videoxogos elaborados en Galicia (Tesis doctoral). Universidade de Santiago de Compostela, Santiago de Compostela.

Martínez, J., Martínez, F.J. y López, R. (2012). Portales educativos españoles: revisión y análisis del uso de servicios Web 2.0. En Investigación bibliotecológica, 26(58), 47-69.

Montero, L. y Vez, X.M. (2003). A elaboración de materiais curriculares como oportunidade para o desenvolvemento profesional do profesorado. En X. Rodríguez (coord.). Materais curriculares e diversidade sociocultural en Galicia. Santiago de Compostela: Concello de Santiago/Nova Escola Galega.

Parcerisa, A. (1996). Materiales curriculares: cómo elaborarlos, seleccionarlos y usarlos. Barcelona: Graó.

Peirats, J., Eirín, R. y Rodríguez, J. (2019). Visiones de los agentes implicados en el diseño, difusión y utilización de los materiales digitales educativos en portales educativos españoles. Educar em Revista, 35(77), 37-58. https://doi.org/10.1590/01044060.68386

Pinto, M. (2015). Calidad y evaluación de los contenidos electrónicos. Electronic Content Management Skills. Recuperado de https://bit.ly/3ojTOh7

Richaudeau, F. (1981). Concepción y producción de manuales escolares. Guía práctica. París: UNESCO.

Sanabria, A., Álvarez, Q. y Peirats, J. (2017). Las políticas educativas en la producción y distribución de materiales didácticos digitales. RELATEC: Revista Latinoamericana de Tecnología Educativa, 16(2), 63-77. http://dx.medra.org/10.17398/1695288X.16.2.63

Sánchez-Jara, J.M., y Vega, A.M. (2019) Nativos digitales, textos digitalizados e impresos mejorados: hacia una transición digital en los recursos para la enseñanza y el aprendizaje. En Ministerio de Educación y Formación Profesional CNIIE. Lectoescritura digital. Madrid: Ministerio de Educación y Formación Profesional. Accesible en https://bit.ly/2JAqp82

Santana, P., Eirín, R. y Marín, D. (2017). Análisisy evaluación de portales institucionales en España. Los casos de Canarias, Galicia y Valencia. RELATEC: Revista Latinoamericana de Tecnología Educativa, 16(2), 29-48. http://doi.org/10.17398/1695-288X.16.2.29

Strauss, A. y Corbin, J. (1998). Basics of qualitative research: Techniques and procedures for developing grounded theory. Thousand Oaks, CA: Sage. 